\title{
Hexadecadienoic Acid n-4
}

National Cancer Institute

\section{Source}

National Cancer Institute. Hexadecadienoic Acid n-4. NCI Thesaurus. Code C68373.

A polyunsaturated long-chain fatty acid with a 16-carbon backbone and double bonds

originating from the 4 th position and either the 6 th or 7 th positions from the methyl end.

At least 2 isomers can be called by this name. 